\title{
Whole-Brain Adaptive 70-kVp Perfusion Imaging with Variable and Extended Sampling Improves Quality and Consistency While Reducing Dose
}

\author{
I. Corcuera-Solano, A.M. McLellan, A.H. Doshi, P.S. Pawha, and L.N. Tanenbaum
}

\begin{abstract}
BACKGROUND AND PURPOSE: Despite common use of CTP to assess cerebral hemodynamics in the setting of ischemia, concerns over radiation exposure remain. Our aim was to evaluate the efficacy of an adaptive 70-kVp (peak) whole-brain CTP protocol with variable sampling intervals and extended duration against an established fixed-sampling, limited-period protocol at $80 \mathrm{kVp}$.
\end{abstract}

MATERIALS AND METHODS: A retrospective analysis of 37 patients with stroke scanned with conventional $(n=17)$ and variant-protocol $(n=20)$ whole-brain CTP was performed. We compared radiation dose, parametric map quality, and consistency of full-contrast circulation capture between a modified 70-kVp protocol, with 20 whole-brain passes at variable sampling intervals over an extended sampling period, and a conventional $80-k V p$ CTP examination with 24 passes at fixed-sampling intervals and a more limited scanning window. Mann-Whitney $U$ test analysis was used to compare both protocols.

RESULTS: The 70-kVp CTP scan provided superior image quality at a $45 \%$ lower CT dose index volume and $13 \%$ lower dose-length product/effective dose compared with the conventional $80-\mathrm{kVp}$ scan. With respect to the consistency of contrast-passage capture, $95 \%$ of the adaptive, extended protocol continued through the venous return to baseline, compared with only $47 \%$ by using the conventional limited-length protocol. Rapid sampling during the critical arterial arrival and washout period was accomplished in nearly $95 \%$ with both the variable and fixed-sampling-interval protocols.

CONCLUSIONS: Seventy-kilovolt (peak) CTP with variable and extended sampling produces improved image quality at lower radiation doses with greater consistency of full contrast passage capture.

ABBREVIATIONS: $\mathrm{CTDI}_{\text {vol }}=\mathrm{CT}$ dose index volume; $\mathrm{DLP}=$ dose-length product

$C^{1}$ perfusion studies involve repeated dynamic scanning during the passage of contrast to and through the brain. The derived parametric maps of $\mathrm{CBF}, \mathrm{CBV}$, and transit times can be integral to the evaluation and management of acute stroke and

Received March 19, 2014; accepted after revision May 16.

From the Department of Neuroradiology, Mount Sinai Medical Center, One Gustave L. Levy Place, New York, New York.

I. Corcuera-Solano and A.M. McLellan contributed equally to this work.

Idoia Corcuera-Solano was supported by a grant from the Spanish foundation Fundación Alfonso Martín Escudero.

Paper previously presented as an electronic exhibit at: Annual Meeting of the European Society of Radiology, March 6-10, 2014; Vienna, Austria; and Annual Meeting of the American Roentgen Ray Society, May 4-9, 2014; San Diego, California; and as an oral presentation at Annual Meeting of the American Society of Neuroradiology and the Foundation of the ASNR Symposium, May 17-22, 2014; Montreal, Quebec, Canada.

Please address correspondence to Lawrence N. Tanenbaum, MD, Mount Sinai Medical Center, Department of Radiology, B1234, One Gustave L. Levy Place, New York, NY 10029; e-mail: nuromri@gmail.com; @nuromri

- Indicates open access to non-subscribers at www.ajnr.org

http://dx.doi.org/10.3174/ajnr.A4043 chronic neurovascular steno-occlusive disease. Despite widespread use, concerns over the potential dangers of high radiation exposure remain, ${ }^{1,2}$ particularly with more recent $4 \mathrm{D}$ or shuttle whole-brain scanning approaches.

Radiation dose is disproportionately dependent on tube voltage, thus the use of lower kilovolt (peak) values has been the principal strategy for managing exposure in CTP. ${ }^{3}$ Additional strategies include reducing the temporal resolution ${ }^{4-6}$ and limiting the scan duration. ${ }^{7}$ Traditionally, CTP studies have been performed at $80 \mathrm{kVp},{ }^{3}$ the lowest energy setting available on most clinical scanners, with a fixed, limited scanning period (40-60+ seconds) and regular sampling intervals (every $1-4$ seconds). ${ }^{4-6}$ We evaluated radiation dose, image quality, and contrast cycle capture consistency of a variant protocol for whole-brain CTP using 3 innovations - lower $70 \mathrm{kVp}$, variable sampling intervals, and longer sampling periods_-comparing it with the existing, conventional approach used at our institution. We hypothesized that the new $70-\mathrm{kVp}$ protocol would result in a reduced radiation dose while providing comparable parametric image quality and improved consistency of full contrast cycle capture 
$70 \mathrm{kv}$ PCT protocol

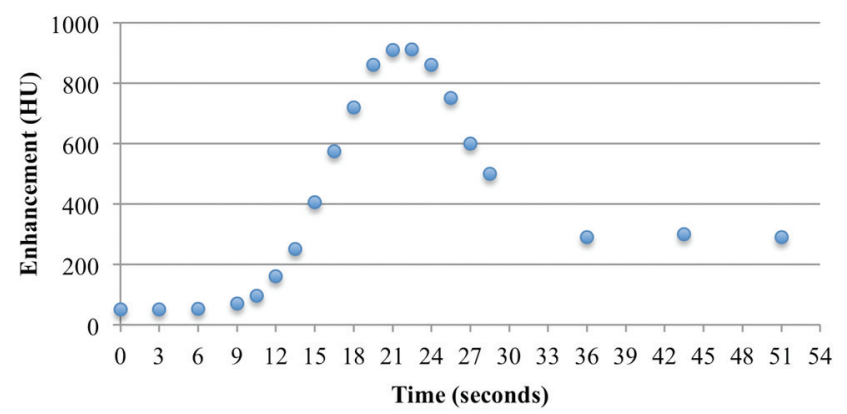

$80 \mathrm{Kv}$ PCT protocol

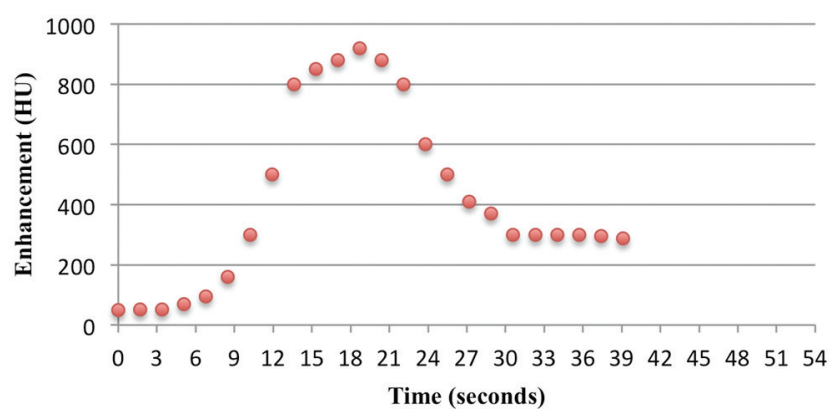

FIG 1. Sample middle-brain time-attenuation curves for the 2 CTP protocols.

compared with conventional approaches. To our knowledge, the use of 70-kVp CTP coupled with variable and extended sampling has not been reported.

\section{MATERIALS AND METHODS \\ Patients}

The institutional review board approved this retrospective single-center, Health Insurance Portability and Accountability Act-compliant study, with a waiver of informed consent. Our institutional radiology data base was queried to identify patients with a presumptive diagnosis of stroke who underwent clinically indicated head CTP from January 2013 through December 2013.

\section{Scanning Technique}

In our institution, patients with suspected acute stroke who are candidates for thrombolysis undergo unenhanced CT. When appropriate, CTP examinations then follow on one of our 2 capable scanners. Patients scanned on our AS+ 128 scanner (Siemens, Erlangen, Germany) are evaluated with our $70-\mathrm{kVp}$ adaptive $4 \mathrm{D}$ spiral CTP protocol. Patients scanned on our LightSpeed VCT (GE Healthcare, Milwaukee, Wisconsin) scanner have a conventional design, helical shuttle $80-\mathrm{kVp}$ examination. Because of scanner limitations, only the $80-\mathrm{kVp}$ examinations benefited from iterative reconstruction (Adaptive Statistical Iterative Reconstruction level 70). The 70-kVp studies were reconstructed with filtered back-projection. All CTPs are performed by using a contrast bolus of $40 \mathrm{~mL}$ of nonionic iodinated contrast media (iopamidol, Isovue 370; Bracco Diagnostics, Princeton, New Jersey) administered via a power injector at a rate of $4 \mathrm{~mL} / \mathrm{s}$, followed by a saline flush of $40 \mathrm{~mL}$ at a $3-\mathrm{mL} / \mathrm{s}$ injection rate. Scanning is initiated 8 seconds after the onset of contrast injection in all cases. In the conventional $80-\mathrm{kVp}$ protocol, 24 whole-brain passes are made at a fixed rate of 1.7 seconds per pass (temporal resolution, 1.7-3.4 seconds in a shuttle mode) for 39 seconds. In the adaptive $70-\mathrm{kV}$ protocol, 20 passes are performed at the following varied intervals: 3 passes every 3 seconds intended for the prearrival baseline, 13 passes every 1.5 seconds targeting the rise and fall of enhancement, and 3 passes at 7.5-second intervals to complete and extend the temporal sampling window for 51 seconds (Fig 1). Detailed technical parameters of the 2 different CTP examinations are given in Table 1 . We routinely extract physiologic, timeresolved CTA from all CTP datasets, avoiding the radiation and contrast needed for an additional dedicated study.
Table 1: CTP parameters

\begin{tabular}{lll}
\hline & \multicolumn{1}{c}{$\mathbf{7 0 ~} \mathbf{~ V \mathbf { p }}$} & \multicolumn{1}{c}{$\mathbf{8 0 ~ \mathbf { ~ V p }}$} \\
\hline Tube current & $150 \mathrm{mAs}$ & $80 \mathrm{mAs}$ \\
Section thickness & $5 \mathrm{~mm}$ & $5 \mathrm{~mm}$ \\
Passes & 20 passes & 24 passes \\
Range & $144 \mathrm{~mm}$ & $120 \mathrm{~mm}$ \\
Time per pass & $3,1.5,7.5$ seconds & 1.7 seconds \\
Scan window & 51 seconds & 39 seconds \\
\hline
\end{tabular}

\section{Data Processing}

All CTP studies were processed at 5-mm thickness with CT Perfusion 4D on an Advantage Workstation 4.6 (GE Healthcare). Arterial and venous input functions were automatically selected by the perfusion software (Perfusion 4D; GE Healthcare) and varied by case, though the algorithm typically defaults to the proximal internal carotid or basilar artery and the sigmoid sinus. Fully automated processing was used unless visual validation by a neuroradiologist suggested the need for semiautomated processing after correction of the arterial and/or venous input over the basilar or internal carotid artery and sigmoid sinus, respectively.

\section{Image Quality Analysis}

All CTP studies were reviewed as saved DICOM datasets on the Advantage 4.6 Workstation.

For quantitative analysis of the parametric images, identical single regions of interest measuring $5 \mathrm{~mm}^{2}$ were placed in the WM and GM of the medial left occipital lobe on the CBF, CBV, and time-to-maximum maps for both CTP protocols. The medial occipital lobe was chosen because it is less likely to be involved in ischemia than the larger MCA territory. If the left posterior cerebral territory was involved, the region of interest was placed on the contralateral normal side. In addition, single ROI measurements were made on source images at the level of the left thalamus at peak arterial and venous enhancement (Fig 2).

We acquired the following quantitative parameters:

1) Signal $(S)$, defined as the mean CT attenuation values in Hounsfield units.

2) Image noise $(I N)$, defined as the $\mathrm{SD}$ of $\mathrm{CT}$ attenuation values.

3) $\mathrm{SNR}=($ Mean WM Region of Interest $) /($ SD WM Region of Interest) or $(S) /(I N)$.

Additionally, 3 radiologists with 6, 9, and 20+ years of neuroradiology experience independently performed a qualitative as- 


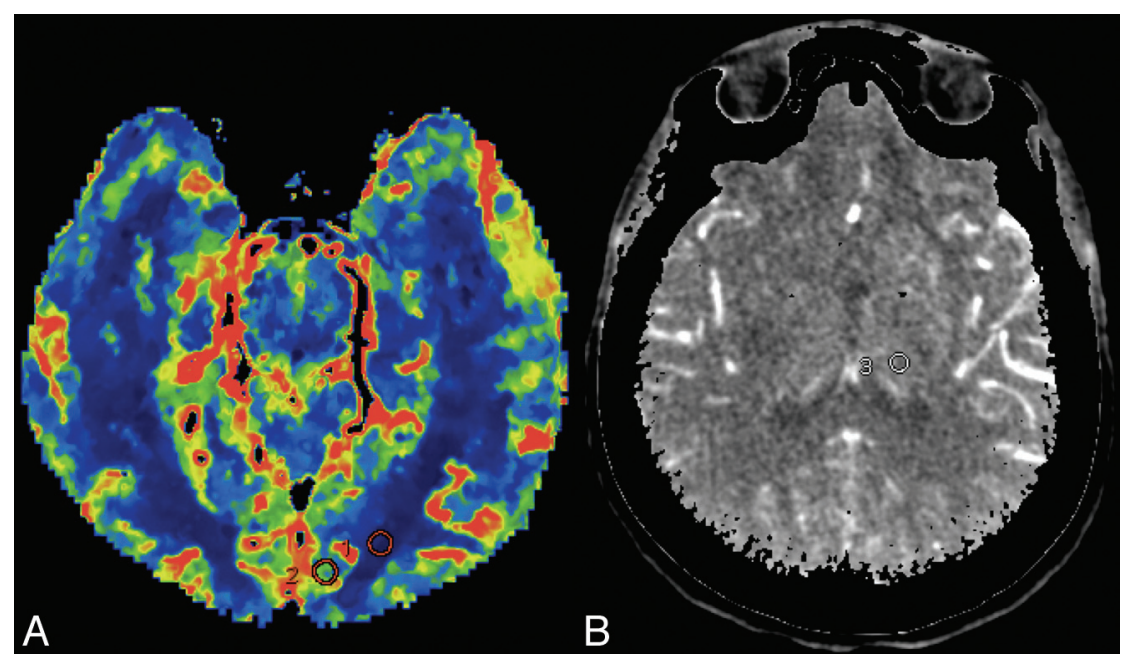

FIG 2. A, Parametric map showing placement of regions of interest in the WM and GM of the left medial occipital lobe. $B$, The region of interest is measured at the peak arterial phase over the left thalamus.

Table 2: Quantitative analysis ${ }^{\mathrm{a}}$

\begin{tabular}{|c|c|c|c|}
\hline & $70 \mathrm{kVp}$ & 80 kVp & $P$ Value \\
\hline \multicolumn{4}{|l|}{ CBF } \\
\hline \multicolumn{4}{|l|}{ WM } \\
\hline Mean & 5.47 & 8.76 & .05 \\
\hline SD & 2.26 & 4.47 & .35 \\
\hline SNR & 2.81 & 3.20 & .66 \\
\hline \multicolumn{4}{|l|}{ GM } \\
\hline Mean & 40.03 & 40.76 & .87 \\
\hline SD & 14.84 & 16.94 & .94 \\
\hline SNR & 4.38 & 3.95 & .92 \\
\hline \multicolumn{4}{|l|}{ CBV } \\
\hline \multicolumn{4}{|l|}{ WM } \\
\hline Mean & 1.50 & 0.95 & .44 \\
\hline SD & 0.41 & 0.35 & .21 \\
\hline SNR & 3.15 & 4.97 & .07 \\
\hline \multicolumn{4}{|l|}{ GM } \\
\hline Mean & 4.88 & 3.49 & .75 \\
\hline SD & 1.45 & 1.08 & .21 \\
\hline SNR & 5.34 & 6.53 & .18 \\
\hline \multicolumn{4}{|l|}{ Tmax } \\
\hline \multicolumn{4}{|l|}{ WM } \\
\hline Mean & 7.81 & 6.52 & .10 \\
\hline SD & 1.72 & 1.63 & .47 \\
\hline SNR & 6.22 & 7.08 & .96 \\
\hline \multicolumn{4}{|l|}{ GM } \\
\hline Mean & 3.94 & 4.51 & .13 \\
\hline SD & 0.68 & 1.03 & .31 \\
\hline SNR & 6.82 & 6.72 & .82 \\
\hline \multicolumn{4}{|l|}{ Arterial max } \\
\hline \multicolumn{4}{|l|}{ Thalamus } \\
\hline Mean & 59.71 & 50.25 & .0005 \\
\hline SD & 11.96 & 11.34 & .426 \\
\hline SNR & 6.43 & 4.69 & .3316 \\
\hline \multicolumn{4}{|l|}{ Venous max } \\
\hline \multicolumn{4}{|l|}{ Thalamus } \\
\hline Mean & 56.72 & 48.68 & .0032 \\
\hline SD & 11.67 & 10.91 & .3811 \\
\hline SNR & 5.11 & 4.62 & .4738 \\
\hline
\end{tabular}

Note:-max indicates maximum; Tmax, time-to-maximum.

${ }^{a}$ Data are presented as mean, SD, and SNR.

sessment of the parametric image sets blinded to the acquisition protocol. Readers were asked to rate overall image quality and gray matter-white matter differentiation of the CBV, CBF, and a variety of transit times maps, including time-to-maximum, time- to-peak, first moment (impulse residual function at the zero time point), and mean transit time on a 3 -point scale: 3 being ideal, 2 being less than ideal, and 1 being nondiagnostic. Window and level settings were standardized for initial review, but each reader was also allowed to vary the settings. Image-quality scores were averaged across readers for analysis and are presented as mean value \pm SD.

\section{Time-Attenuation Curve Analysis}

A board-certified radiologist with 1 year of neuroradiology experience analyzed and compared the first-pass perfusion time-attenuation curves to assess the rate at which each protocol imaged the desired portions of the enhancement cycle as intended: capture of the precontrast arrival baseline, fast sampling from the arterial contrast arrival through the midportion of the downslope back to baseline, and continued sampling through the venous return to baseline.

\section{Radiation Dose Assessment}

Mean doses of the 2 protocols were recorded and compared. The CT dose index volume $\left(\mathrm{CTDI}_{\mathrm{vol}}\right)$ in milligrays and the doselength product (DLP) were extracted from the scan dose page. The effective dose in millisieverts was estimated by multiplying the DLP by a constant region-specific conversion coefficient of $0.0023 \mathrm{mSv} /(\mathrm{mGy} \cdot \mathrm{cm}){ }^{8}$ The percentage reduction when comparing the mean $\mathrm{CTDI}_{\mathrm{vol}}$ used in examinations with $80 \mathrm{kVp}$ with the mean $\mathrm{CTDI}_{\mathrm{vol}}$ of $70 \mathrm{KVp}$ was calculated according to the following formula: $100 \%-\left(\mathrm{CTDI}_{\mathrm{vol}} 70 \mathrm{kVp} / \mathrm{CTDI}_{\mathrm{vol}} 80\right.$ $\mathrm{kVp}) \times 100$. The same analysis was performed with the DLP and effective dose.

\section{Statistical Analysis}

Statistical analyses were performed by using the commercial GraphPad Prism, Version 5.00 for Mac OS X (GraphPad Software, San Diego California). Radiation variables were compared by using the Student $t$ test for unpaired samples. The Kendall coefficient of concordance was calculated to evaluate the degree of consensus among the 3 observers. ${ }^{9}$ The Mann-Whitney $U$ test was applied to compare image-quality results between the $70-\mathrm{kVp}$ and $80-\mathrm{kVp}$ CTP protocols. Numeric data were expressed as mean \pm SD. $P<.05$ was a statistically significant result.

\section{RESULTS}

Thirty-seven patients ( 15 men and 22 women), with a mean age of 66 years (range, 48-92 years) and a presumptive diagnosis of stroke, underwent brain CTP in our institution. Of those, 17 cases were performed with the conventional $80-\mathrm{kVp}$ CTP protocol (4 men and 13 women; mean age, 64 years), and 20, with the adaptive $70-\mathrm{kVp}$ CTP ( 9 men and 11 women; mean age, 66 years). The average NIHSS score was 9.35 for the patients scanned with the $70-\mathrm{kVp}$ and 12.06 for those scanned with the $80-\mathrm{kVp}$ protocol. 
The overall incidence of stroke was $70.3 \%$ (26/37), with $88.5 \%$ $(23 / 26)$ involving the anterior circulation and $11.5 \%(3 / 26)$, the posterior circulation. Fourteen stroke cases were diagnosed by using 70-kVp protocol: Eleven were anterior circulation strokes (5 left MCA, 1 left ICA, and 5 right MCA), and 3 were posterior strokes, involving the pons, right middle cerebellar peduncle, and bilateral cerebellum. Twelve stroke cases were diagnosed with the 80-kVp protocol: All were anterior strokes, with 7 left MCA, 1 left ICA, 2 right MCA, 1 right anterior cerebral artery/MCA borderzone, and 1 right anterior choroidal artery.

The time from symptom onset to scanning ranged from 25 minutes to 5 hours in the $70-\mathrm{kVp}$ group and 50 minutes to 4 hours at $80 \mathrm{kVp}$. A stroke alert response time of $<20$ minutes to CT was maintained in all patients who presented on arrival to the emergency department.

Table 3: Qualitative analysis of overall quality and gray-white matter differentiation in $80-k V p$ and $70-k V p$ whole-brain CTP $^{a}$

\begin{tabular}{|c|c|c|c|}
\hline & $70 \mathrm{kVp}$ & $80 \mathrm{kVp}$ & $P$ Value \\
\hline \multicolumn{4}{|c|}{ Overall quality } \\
\hline CBF & $2.95 \pm 0.167$ & $2.81 \pm 2.84$ & .0459 \\
\hline CBV & $2.95 \pm 0.167$ & $2.76 \pm 0.305$ & .0173 \\
\hline $\mathrm{TT}$ & $2.89 \pm 0.224$ & $2.69 \pm 0.276$ & .0113 \\
\hline \multicolumn{4}{|c|}{ GM-WM differentiation } \\
\hline $\mathrm{CBF}$ & $2.91 \pm 0.187$ & $2.83 \pm 0.314$ & .5598 \\
\hline CBV & $2.93 \pm 0.178$ & $2.70 \pm 0.347$ & .0278 \\
\hline $\mathrm{TT}$ & $2.86 \pm 0.231$ & $2.69 \pm 0.276$ & .0630 \\
\hline
\end{tabular}

Note:-TT indicates transit time.

${ }^{a}$ Data are presented as means and SD.

\section{Image Quality}

No significant quantitative differences were found on parametric maps between protocols. Source image contrast enhancement was significantly higher at $70 \mathrm{kVp}$ than with $80 \mathrm{kVp}$, though no significant differences in noise or SNR were found. Quantitative data are summarized in Table 2. The interobserver agreement in the qualitative assessment of parametric maps was good (Kendall coefficient, 0.46). ${ }^{9}$ Overall image quality was significantly better by using the $70-\mathrm{kVp}$ CTP protocol for all 3 parameters: $\mathrm{CBF}(P=$ $.02), \mathrm{CBV}(P=.04)$, and transit times $(P=.01)$, compared with $80 \mathrm{kVp}$. Scores of the $70-\mathrm{kVp}$ and $80-\mathrm{kVp}$ CTP protocols for GM-WM differentiation were comparable in $\mathrm{CBF}$ and transit times, while CBV using 70-kVp CTP was associated with significantly better GM-WM differentiation $(P=.02)$ (Table 3 and Figs 3 and 4).

\section{Time-Attenuation Curves}

Time-attenuation curve analysis revealed that by using the adaptively sampled longer 70-kVp CTP technique (sampling extended to 51 seconds), $95 \%(19 / 20)$ of the studies captured the venous return to baseline, compared with only $47 \%(8 / 17)$ of the studies scanned by using the fixed-interval limited-length $80-\mathrm{kVp}$ protocol (39 seconds). Rapid sampling during the critical arterial arrival and washout period was comparable in both protocols, captured at $95 \%(19 / 20)$ with the $70-\mathrm{kVp}$ adaptive protocol and at $94 \%(16 / 17)$ with the $80-\mathrm{kV}$ fixed protocol.

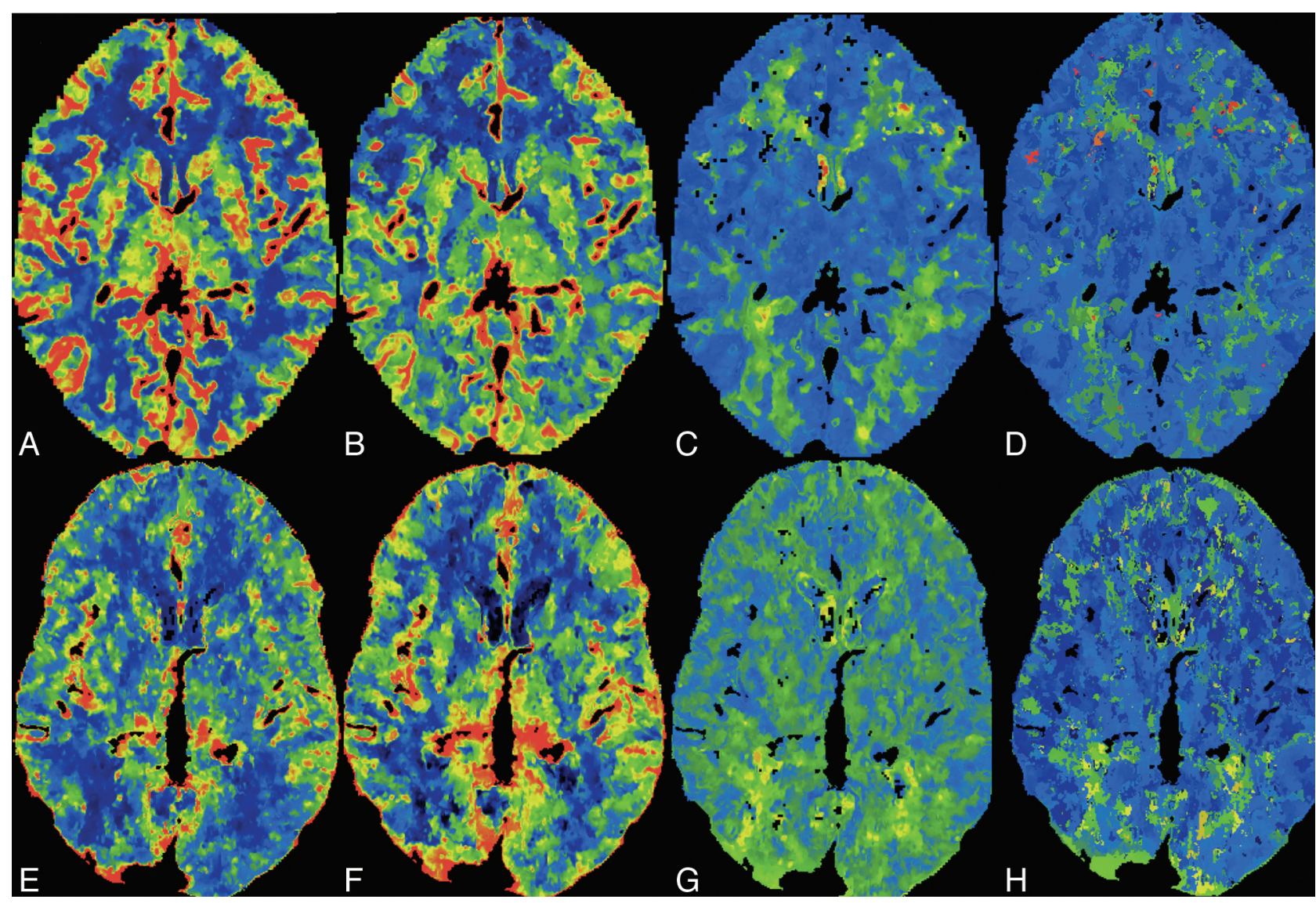

FIG 3. Normal-sample CTP datasets from the $70-k V p$ protocol: $C B F(A), C B V(B)$, time-to-maximum (C), TTP (D). From the 80-kVp protocol: $C B F$ $(E)$, CBV $(F)$, time-to-maximum $(G)$, TTP $(H)$. 


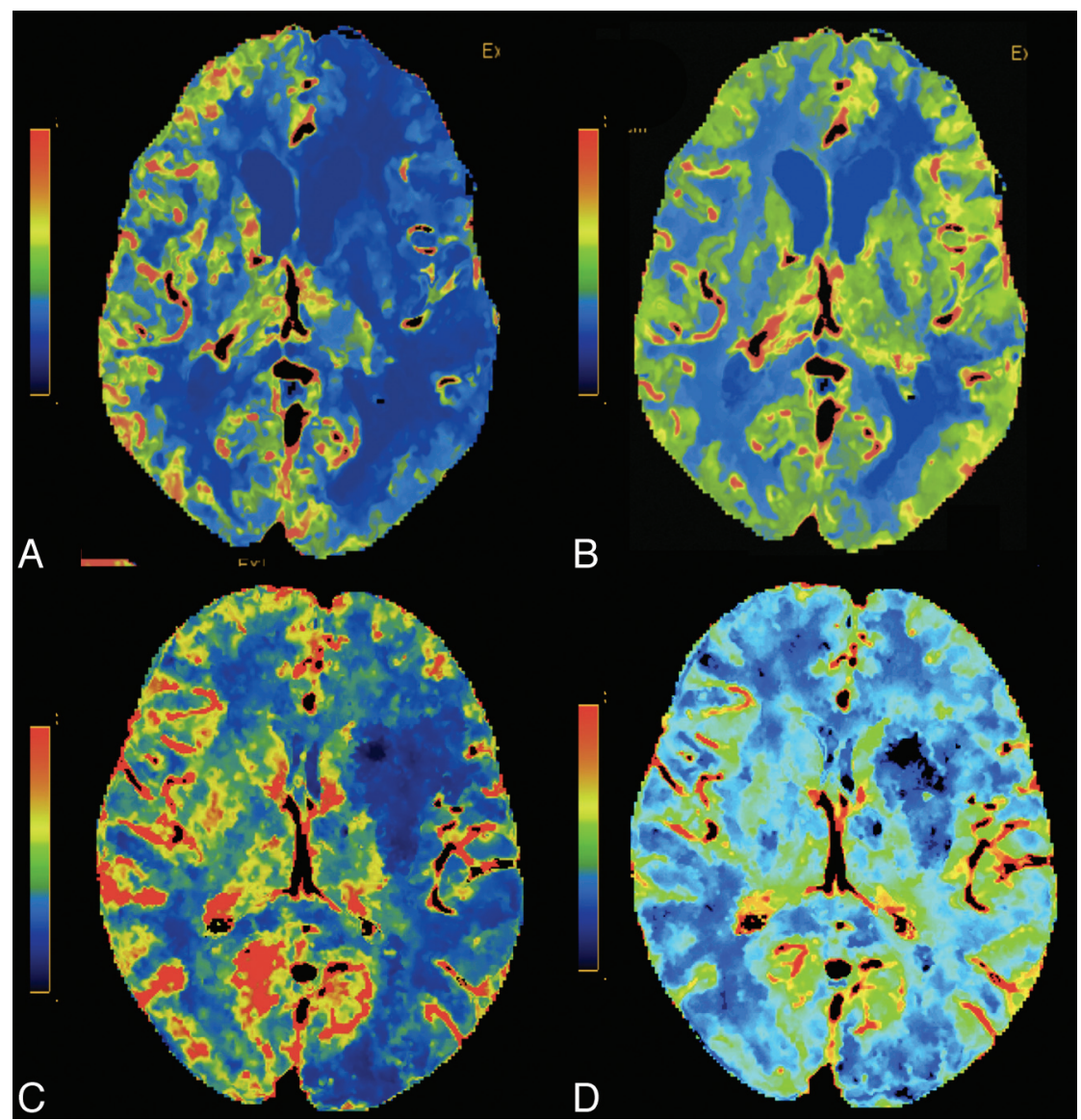

FIG 4. Two different patients with left $M C A$ ischemia. $C B F(A)$ and $C B V(B)$ at 70-kVp and $C B F(C)$ and CBV $(D)$ at $80-k V p$ CTP. Note the decreased CBF and preserved CBV.

Table 4: CTDI $_{\text {vol }}$ and DLP/ED values for whole-brain CTP examinations at $80 \mathrm{kVp}$ and $70 \mathrm{kVp}$ with different scanners

\begin{tabular}{|c|c|c|c|}
\hline & \multirow[b]{3}{*}{$70 \mathrm{kVp}$} & \multirow[b]{3}{*}{$80 \mathrm{kVp}$} & \multirow{2}{*}{$\frac{\text { Percentage Reduction }}{100 \%-(70 \mathrm{kVp} / 80 \mathrm{kVp}) \times}$} \\
\hline & & & \\
\hline & & & 100 \\
\hline $\mathrm{CTDI}_{\mathrm{vol}}(\mathrm{mGy})$ & 105 & 192 & 45.31 \\
\hline $\mathrm{DLP}(\mathrm{mGy} \cdot \mathrm{cm})$ & 1588 & 1831 & 13.27 \\
\hline $\mathrm{ED}(\mathrm{mSv})$ & 3.65 & 4.21 & 13.27 \\
\hline
\end{tabular}

Note:-ED indicates effective dose.

\section{Radiation Dose}

Differences in mean CTDI $_{\text {vol }}$, DLP, and effective dose between groups were statistically significant (all $P<.01$ ). At $70 \mathrm{kVp}$, there was a $45.31 \%$ reduction of the mean $\mathrm{CTDI}_{\mathrm{vol}}(105 \mathrm{mGy})$ compared with $80 \mathrm{kVp}(192 \mathrm{mGy})$. Despite an approximately 12-second wider scanning window and greater anatomic coverage (144 versus $120 \mathrm{~mm}$ ), there was a $13.27 \%$ reduction of the mean DLP $\left(1588 \mathrm{mGy}^{\circ} \mathrm{cm}\right)$ and of the mean effective dose $(3.65 \mathrm{mSv})$ when using the $70-\mathrm{kVp}$ protocol compared with the $1831 \mathrm{mGy} \cdot{ }^{\circ} \mathrm{cm}$ and $4.21 \mathrm{mSv}$ at $80 \mathrm{kVp}$. Table 4 summarizes the radiation dose results.

\section{DISCUSSION}

In this study, we evaluated the efficacy of a $70-\mathrm{kVp}$ whole-brain CTP protocol with variable sampling intervals and extended sampling against our established fixed-sampling, limited-period protocol at $80 \mathrm{kVp}$. Our protocol goals included reduced dose, matched or superior image quality, and more consistent contrast- passage capture. To the best of our knowledge, our protocol is an approach that has not yet been reported.

Several methods have been proposed to limit the radiation dose in CTP, including reducing the tube voltage, ${ }^{3,10,11}$ lowering the tube current, ${ }^{10}$ dropping the temporal resolution, ${ }^{4-6}$ and shortening the scan duration. ${ }^{7}$ Reducing the tube voltage has a disproportionate effect on dose (eg, there is a 5-fold dose reduction in shifting from 140 to $80 \mathrm{kVp}$ ). ${ }^{12}$ Wintermark et $\mathrm{al}^{3}$ demonstrated that moving to $80 \mathrm{kVp}$ from $120 \mathrm{kVp}$ both reduced radiation dose, reporting a CTDI ${ }_{\text {vol }}$ of 291 $\mathrm{mGy}$, and increased the effectiveness of contrast enhancement in CTP. At present, the widely practiced standard for CTP has been $80 \mathrm{kVp}$ at $200 \mathrm{mAs}$ or less. ${ }^{3,13}$ Our study used $70 \mathrm{kVp}$, a setting available on many clinical scanners, and $150 \mathrm{mAs}$, resulting in a $\mathrm{CTDI}_{\mathrm{vol}}$ of $105 \mathrm{mGy}, 63 \%$ lower than the $80-\mathrm{kVp}$ protocol used in the study of Wintermark et al. ${ }^{3}$ The use of even lower kilovolt (peak) further enhances the attenuation of contrast because the $70-\mathrm{kV}$ p beam energy even more closely approximates the iodine k-edge $(33.2 \mathrm{KeV}) .{ }^{12}$ The net result is a lower net dose per whole-brain pass.

Another approach to radiation dose limitation is the use of wider sampling intervals. Conventional CTP studies use regular sampling intervals for a fixed period with length limited by dose concerns. The optimal sampling rate remains controversial. Wintermark et $\mathrm{al}^{4}$ found that a sampling interval of $>1$ second could be used without altering the quantitative accuracy of CTP and recommended a sampling interval of 3 seconds over 42 seconds if a contrast bolus of $40 \mathrm{~mL}$ with an injection rate of $4 \mathrm{~mL} / \mathrm{s}$ is used. Wiesmann et al ${ }^{14}$ also stated that temporal resolution could be reduced to 3 seconds without significant compromise in image quality. In a more recent study, Shankar et $\mathrm{al}^{15}$ suggested the use of a temporal resolution of up to 4 seconds for whole-brain CTP. However, other groups concluded that sampling intervals longer than 1 second yielded significantly poorer depiction of ischemic areas. ${ }^{5,6,16}$

Traditional fixed-sampling approaches have a uniform scanning rate over the entire CTP acquisition. Because certain portions of the contrast passage are likely less sensitive to sampling frequency, such as the pre-enhancement baseline and the downward slope toward the return to and through baseline, reducing sampling rates during these segments is appealing. The "omitted" samples can be traded for dose reduction or for higher temporal resolution during key segments of arterial tissue passage. Variable sampling for CTP has been reported by investigators working with wide 320-channel detector systems with favorable results. ${ }^{15}$ To date, variable intervals have yet to be investigated on the much 
more commonly used conventional 64-channel detector systems, despite wide clinical availability.

Overall scanning time can also be reduced in the interest of dose control. With fixed-sampling, the dose is directly proportional to the scanning period, which encourages shorter scans. Shorter scan time increases the risk that due to variability in cardiac output, a number of examinations may not continue through the venous return to baseline; this difference challenges the calculation of parametric maps based on deconvolution principles. ${ }^{17,18}$ Longer sampling periods provide greater consistency of capture of the full contrast cycle from arterial arrival through venous return over a broad range of cardiac outputs. Adaptive asymmetric sampling intervals permit extended scan windows with dose neutrality because scans ordinarily taken at close intervals during before, and after arterial passage are traded for those at wide intervals at the end of the scanning period; the trading affords protection against premature termination. With a variable sampling rate, dose is independent of the length of the scanning window and instead is related to the total number of samples.

The classic CTP protocol described by Wintermark et $\mathrm{al}^{4}$ had only a 40-second scanning window, starting 7 seconds after the contrast injection, similar to the 39-second and 8-second delay for the 24 fixed-rate whole-brain passes of our standard $80-\mathrm{kVp}$ examination. In the variable-rate $70-\mathrm{kV}$ p protocol, the scan window for 20 whole-brain passes was extended to 51 seconds. Analysis of the time-attenuation curve revealed that when scanning with the wider CTP window, nearly all of the studies continued through the venous return to baseline, while only the half of the studies using the limited-length protocol did. Discovery of this failure rate provoked a subsequent change in our clinical $80-\mathrm{kVp}$ protocol, with the sampling time now extended to 43 seconds at the cost of a modest $10 \%$ increase in dose.

Previous studies using whole-brain CTP imaging with fixedsampling rates reported radiation doses of $7.6 \mathrm{mSv},{ }^{19} 4.56 \mathrm{mSv},{ }^{20}$ and $4.6 \mathrm{mSv},{ }^{21}$ depending on the protocol. With our 20 wholebrain passes at $70 \mathrm{kVp}$, we achieved a considerable reduction of the effective dose to $3.65 \mathrm{mSv}$. Despite a $13 \%$ reduction in the effective dose compared with our standard $80-\mathrm{kVp}$ protocol, overall quality was rated significantly better in the $70-\mathrm{kVp}$ protocol and GM-WM differentiation was similar in both the quantitative and qualitative analyses.

We acknowledge several limitations in our study. The retrospective study design and small sample size may have introduced sampling errors or other dataset biases and require a confirmation of our findings in a prospective trial and a larger population. In addition, we compared studies between scanners from different manufacturers with different acquisition methods. We are unable to exclude the possible influence of different technical specifications such as $\mathrm{x}$-ray generator/tube, detection system, and reconstruction algorithm on image quality and radiation dose. Parameters such as z-axis coverage of each acquisition, detector configuration, and the use of shuttle mode are slightly different between scanner models. Finally, while all the parametric maps were processed with a form of $4 \mathrm{D}$ noise reduction, because of scanner limitations, the $70-\mathrm{kVp}$ datasets did not benefit from iterative reconstruction as did the CTP studies at $80 \mathrm{kVp}$; this difference diminished the impact of protocol variation on image quality and possible dose reduction. A future study might seek to validate our conclusions on a single scanner.

\section{CONCLUSIONS}

Whole-brain adaptive perfusion imaging at $70 \mathrm{kVp}$ produces significant improvement in image quality compared with traditional $80-\mathrm{kVp}$ acquisitions at lower radiation doses while providing greater consistency of full-contrast transit capture.

Disclosures: Idoia Corcuera-Solano_UNRELATED: Grant: Fundación Alfonso Martín Escudero, Comments: The foundation paid my salary as a research fellow in Mount Sinai when I was conducting research for this article. Lawrence N. TanenbaumUNRELATED: Payment for Lectures (including service on Speakers Bureaus): Siemens, GE Healthcare.

\section{REFERENCES}

1. Ringelstein A, Lechel U, Fahrendorf DM, et al. Radiation exposure in perfusion CT of the brain. J Comput Assist Tomogr 2014;38:25-28

2. Imanishi Y, Fukui A, Niimi H, et al. Radiation-induced temporary hair loss as a radiation damage only occurring in patients who had the combination of MDCT and DSA. Eur Radiol 2005;15:41-46

3. Wintermark $M$, Maeder $P$, Verdun FR, et al. Using $80 \mathrm{kV}$ p versus 120 $\mathrm{kVp}$ in perfusion CT measurement of regional cerebral blood flow. AJNR Am J Neuroradiol 2000;21:1881-84

4. Wintermark M, Smith WS, Ko NU, et al. Dynamic perfusion CT: optimizing the temporal resolution and contrast volume for calculation of perfusion CT parameters in stroke patients. AJNR Am J Neuroradiol 2004;25:720-29

5. Kämena A, Streitparth F, Grieser C, et al. Dynamic perfusion CT: optimizing the temporal resolution for the calculation of perfusion CT parameters in stroke patients. Eur J Radiol 2007;64:111-18

6. Abels B, Klotz E, Tomandl BF, et al. CT perfusion in acute ischemic stroke: a comparison of 2-second and 1-second temporal resolution. AJNR Am J Neuroradiol 2011;32:1632-39

7. Hirata M, Sugawara Y, Murase K, et al. Evaluation of optimal scan duration and end time in cerebral CT perfusion study. Radiat Med 2005;23:351-63

8. Bogartz G. Golding SJ, Jurik AG, et al. European Guidelines for Multislice Computed Tomography. 2004. http://w3.tue.nl/fileadmin/ sbd/Documenten/Leergang/BSM/European_Guidelines_Quality_ Criteria_Computed_Tomography_Eur_16252.pdf. Accessed February 19,2014

9. Siegel S, Castellan JN. Nonparametric Statistics for the Behavioral Sciences. New York: McGraw-Hill; 1988

10. Hirata M, Sugawara $Y$, Fukutomi $Y$, et al. Measurement of radiation dose in cerebral CT perfusion study. Radiat Med 2005;23:97-103

11. Cohnen M, Fischer H, Hamacher J, et al. CT of the head by use of reduced current and kilovoltage: relationship between image quality and dose reduction. AJNR Am J Neuroradiol 2000;21:1654-60

12. Huda W, Lieberman KA, Chang J, et al. Patient size and $\mathbf{x}$-ray technique factors in head computed tomography examinations. II. Image quality. Med Phys 2004;31:595-601

13. Wintermark M, Lev MH. FDA investigates the safety of brain perfusion CT. AJNR Am J Neuroradiol 2010;31:2-3

14. Wiesmann M, Berg S, Bohner G, et al. Dose reduction in dynamic perfusion CT of the brain: effects of the scan frequency on measurements of cerebral blood flow, cerebral blood volume, and mean transit time. Eur Radiol 2008;18:2967-74

15. Shankar JJ, Lum C, Sharma M. Whole-brain perfusion imaging with 320-MDCT scanner: reducing radiation dose by increasing sampling interval. AJR Am J Roentgenol 2010;195:1183-86

16. Kloska SP, Fischer $T$, Sauerland $C$, et al. Increasing sampling interval in cerebral perfusion CT: limitation for the maximum slope model. Acad Radiol 2010;17:61-66

17. Konstas AA, Goldmakher GV, Lee TY, et al. Theoretic basis and tech- 
nical implementations of CT perfusion in acute ischemic stroke. Part 1: Theoretic basis. AJNR Am J Neuroradiol 2009;30:662-68

18. Fieselmann A, Kowarschik M, Ganguly A, et al. Deconvolutionbased CT and MR brain perfusion measurement: theoretical model revisited and practical implementation details. Int J Biomed Imaging 2011;2011:467563

19. Page M, Nandurkar D, Crossett MP, et al. Comparison of $4 \mathrm{~cm}$ z-axis and 16 cm z-axis multidetector CT perfusion. Eur Radiol 2010;20:1508-14
20. Diekmann S, Siebert E, Juran R, et al. Dose exposure of patients undergoing comprehensive stroke imaging by multidetector-row $\mathrm{CT}$ : comparison of 320-detector row and 64-detector row CT scanners. AJNR Am J Neuroradiol 2010;31:1003-09

21. Wintermark M, Maeder P, Thiran JP, et al. Quantitative assessment of regional cerebral blood flows by perfusion CT studies at low injection rates: a critical review of the underlying theoretical models. Eur Radiol 2001;11:1220-30 